\title{
Mutual-exclusion statistics in exactly solvable models in one and higher dimensions at low temperatures
}

\author{
Yasuhiro Hatsugai* and Mahito Kohmoto \\ Institute for Solid State Physics, University of Tokyo, 7-22-1 Roppongi, Minato-ku, Tokyo 106, Japan \\ Tohru Koma ${ }^{\dagger}$ \\ Department of Physics, Gakushuin University, Mejiro, Toshima-ku, Tokyo 171, Japan \\ Yong-Shi Wu \\ Department of Physics, University of Utah, Salt Lake City, Utah 84112 \\ (Received 18 April 1995; revised manuscript received 6 May 1996)
}

\begin{abstract}
We study statistical characterization of the many-body states in exactly solvable models with internal degrees of freedom. The models under consideration include the isotropic and anisotropic Heisenberg spin chains, the Hubbard chain, and a model in higher dimensions which exhibits the Mott metal-insulator transition. It is shown that the ground states of these systems are all described by that of a generalized ideal gas of particles (called exclusons) which have mutual-exclusion statistics, either between different rapidities or between different species. For the Bethe ansatz solvable models, the low-temperature properties are well described by the excluson description if the degeneracies due to string solutions with complex rapidities are taken into account correctly. For the Hubbard chain with strong but finite coupling, charge-spin separation is shown for thermodynamics at low temperatures. Moreover, we present an exactly solvable model in arbitrary dimensions which, in addition to giving a perspective view of spin-charge separation, constitutes an explicit example of mutual-exclusion statistics in more than two dimensions. [S0163-1829(96)00332-3]
\end{abstract}

\section{INTRODUCTION}

Elementary particles or excitations are usually classified either as bosons or as fermions. In recent years, however, it has been recognized that particles with "fractional statistics" intermediate between bosons and fermions can exist in two-dimensional $^{1}$ or one-dimensional ${ }^{2,3}$ systems. In two dimensions, a type of fractional statistics can be defined on the basis of the phase factor $\exp (i \theta)$, with $\theta$ allowed to be arbitrary, associated with an exchange of identical particles. One has $\theta=0$ for bosons and $\theta=\pi$ for fermions. A particle obeying such fractional statistics (with $\theta \neq 0$ or $\pi$ ) is called as "anyon.," It is believed that the quasiparticles and quasiholes in fractional quantum Hall liquids are anyons. ${ }^{5}$ Anyons can exist only in two spatial dimensions due to the braid group structure associated with them. ${ }^{6}$

Another aspect of quantum statistics involves state counting, or the exclusive nature of the particles. Any number of bosons can be in a single-particle quantum state. Therefore there is no exclusion between bosons. On the other hand, the exclusion is perfect for fermions in the sense that a singleparticle state can accommodate at most one fermion. This aspect of quantum statistics can be generalized, as noticed by Haldane, ${ }^{3}$ who proposed a definite generalization of the Pauli principle such that one can consider particles with nonperfect exclusion. He pointed out that a spinon in a one-dimensional long-range interacting quantum spin chain can exclude, on average, half of the other spinons in occupying a singleparticle state. We shall call such a generalization "exclusion statistics" and a particle obeying it an "excluson." In contrast to the usual bosons and fermions, the general concept of exclusons allows mutual statistics; namely, there may exist statistical interactions or mutual exclusion between different species of particles. Haldane has recognized ${ }^{3}$ that quasiparticles in fractional quantum Hall fluids are exclusons with mutual statistics between quasielectrons and quasiholes.

Thus the concepts of fractional anyon statistics and exclusion statistics constitute generalizations of two different aspects (exchange phase and exclusion) of the usual quantum statistics. An essential difference between the two concepts is that anyons can exist only in two spatial dimensions, while in principle exclusons may exist in any dimensions.

Recently, one of us ${ }^{7}$ introduced the concept of a generalized ideal gas of exclusons (see Sec. II for definition), and showed that its thermodynamic properties can be easily understood through a statistical distribution that interpolates between bosons and fermions. Later, Bernard and $\mathrm{Wu}^{8}$ have shown that the Bethe ansatz solvable models in one dimension can be described as an ideal (or noninteracting) excluson gas. This exemplifies that in certain circumstances particle-particle interactions can be totally absorbed by the statistical interactions. (A possible relation to a conformal field theory was discussed by Fukui and Kawakami. ${ }^{9}$ ) Thus the concept of exclusion statistics may become a powerful tool in understanding certain interacting many-body problems. For example, recently it has been shown ${ }^{10}$ that the essential features of low-temperature physics of Luttinger liquids in one dimension can be approximately described by a system of noninteracting exclusons. This may provide a new approach to interacting many-body systems.

The examples considered by Bernard and $\mathrm{Wu}$ are the repulsive $\delta$-function boson gas ${ }^{11}$ and the Calogero-Sutherland model. ${ }^{12}$ Both of them contain only a single species. The present paper is a followup to study statistical interactions or 
mutual statistics in exactly solvable models with internal quantum numbers. We will discuss one-dimensional Bethe ansatz solvable models such as the isotropic $(X X X)$ and anisotropic $(X X Z)$ Heisenberg chains, and the Hubbard chain. In addition, we will consider an exactly solvable model in arbitrary dimensions proposed by two of us, ${ }^{13}$ which has the Mott metal-insulator transition. A common feature in all these models is that there exists mutual exclusion either between particles of different rapidities or between different species.

In this paper we are going to address the following two questions. First, many Bethe ansatz solvable models with internal degrees of freedom allow solutions of the Bethe ansatz equations with complex rapidities, while a naive analysis of the Yang-Yang thermodynamic Bethe ansatz in terms of exclusons deals only with excitations which correspond to solutions with real rapidities. The question is whether these excitations are sufficient to give correct thermodynamic properties at low temperatures or not. Previously, in Ref. 9 the same topic of thermodynamics in terms of exclusons with multiple species was discussed without addressing this problem in the Bethe ansatz solvable models. For the Heisenberg spin chain, we explicitly show that at the isotropic antiferromagnetic point (with the anisotropy parameter $\Delta=1$ ), the generalized ideal excluson gas of excitations with real rapidities does give correct low-temperature behavior if a double degeneracy of the excitations induced by complex rapidities is taken into account. The second question deals with the excluson description for the physically interesting phenomenon of charge-spin separation. Both the Hubbard chain and the exactly solvable model in higher dimensions exhibit this phenomenon under certain conditions. We will show that when this happens indeed the two models can be described by two species (spin and charge) of exclusonic excitations with nontrivial mutual statistics. In particular, the latter model provides an example of (mutual-)exclusion statistics in more than two dimensions. We note that our treatment of the models differs from that presented in Ref. 14.

\section{EXCLUSON DESCRIPTION}

We consider a system with a total number $N=\sum_{j, \mu} N_{j}^{\mu}$ of particles or quasiparticles, where $N_{j}^{\mu}$ is the number of particles of species $\mu$ with a set of good quantum numbers, collectively denoted by $j$, specifying the states. Following Ref. 7, we assume that the total number of states with $\left\{N_{j}^{\mu}\right\}$ is

$$
W=\prod_{i, \mu} \frac{\left[D_{i}^{\mu}\left(\left\{N_{j}^{\nu}\right\}\right)+N_{i}^{\mu}-1\right] !}{N_{i}^{\mu} !\left[D_{i}^{\mu}\left(\left\{N_{j}^{\nu}\right\}\right)-1\right] !},
$$

where $D_{i}^{\mu}\left(\left\{N_{j}^{\nu}\right\}\right)$ is the number of available single-particle states (counted as bosons), which by definition is given by

$$
D_{i}^{\mu}\left(\left\{N_{j}^{\nu}\right\}\right)+\sum_{j, \nu} g_{i j}^{\mu \nu} N_{j}^{\nu}=G_{i}^{\mu},
$$

with statistical interactions $g_{i j}^{\mu \nu}$ Here $G_{i}^{\mu}$ is the number of available single-particle states when there is no particle in the system, namely, $G_{i}^{\mu}=D_{i}^{\mu}(\{0\})$. The derivative of (2.2) is

$$
\frac{\partial D_{i}^{\mu}\left(\left\{N_{j}^{\nu}\right\}\right)}{\partial N_{j}^{\nu}}=-g_{i j}^{\mu \nu},
$$

agreeing with the original definition for "statistical interactions" proposed by Haldane. ${ }^{3}$ When the pair $(i, \mu)$ differs from $(j, \nu)$, we call $g_{i j}^{\mu \nu}$ mutual statistics between particles labeled by $(i, \mu)$ and those by $(j, \nu)$.

We further assume that the total energy of the system with $\left\{N_{j}^{\mu}\right\}$ particles is always simply given by

$$
E=\sum_{j, \mu} N_{j}^{\mu} \epsilon_{j}^{\mu}
$$

with constant $\epsilon_{j}^{\mu}$. Equations (2.1), (2.2), and (2.4) define the generalized ideal gas of exclusons. ${ }^{7}$ It is known that (2.4) is not satisfied for free anyons. ${ }^{15}$ One of us ${ }^{7}$ has derived the statistical distribution for the generalized ideal gas and the thermodynamics following from it. The equilibrium statistical distribution for $\left\{N_{i}^{\mu}\right\}$ is determined by

$$
w_{i}^{\mu} N_{i}^{\mu}+\sum_{j, \nu} g_{i j}^{\mu \nu} N_{j}^{\nu}=G_{i}^{\mu},
$$

where $w_{i}^{\mu}$ satisfy the equations

$$
\left(1+w_{i}^{\mu}\right) \prod_{j, \nu}\left(\frac{w_{j}^{\nu}}{1+w_{j}^{\nu}}\right)^{g_{j i}^{\mu \nu}}=\exp \left[\frac{\epsilon_{i}^{\mu}-a^{\mu}}{T}\right],
$$

where $a^{\mu}$ is the chemical potential for particles of species $\mu$. The thermodynamic potential is given by

$$
\begin{gathered}
\Omega \equiv-T \ln Z \\
=-T \sum_{\mu, i} G_{i}^{\mu} \ln \left[\frac{G_{i}^{\mu}+N_{i}^{\mu}-\sum_{j, \nu} g_{i j}^{\mu \nu} N_{j}^{\nu}}{G_{i}^{\mu}-\sum_{j, \nu} g_{i j}^{\mu \nu} N_{j}^{\nu}}\right],
\end{gathered}
$$

where $Z$ is the grand partition function.

\section{THERMODYNAMIC BETHE ANSATZ}

A large class of models which can be interpreted as the generalized ideal gas is the Bethe ansatz solvable models in one dimension. It is well known that the generalized ideal gas actually represents a system of interacting particles. The Bethe ansatz method can be applied to systems with the special property that all the scattering amplitudes for many quasiparticles can be written in terms of two-body scattering amplitudes. ${ }^{16}$ As a result, the eigenvalue problem of a Bethe ansatz solvable system is reduced to solving the so-called Bethe ansatz equations. ${ }^{17}$

In general, the Bethe ansatz equations may be rewritten as

$$
L p_{\mu}\left(\lambda_{\ell}^{\mu}\right)=2 \pi I_{\ell}^{\mu}+\sum_{m, \nu} \theta_{\mu \nu}\left(\lambda_{\ell}^{\mu}, \lambda_{m}^{\nu}\right),
$$

with $\theta_{\mu \nu}\left(\lambda_{\ell}^{\mu}, \lambda_{m}^{\nu}\right)$ being the two-body scattering phase shift between the quasiparticles with rapidities $\lambda_{\ell}^{\mu}$ and $\lambda_{m}^{\nu}$. Here $L$ is the size of the system, $p_{\mu}\left(\lambda_{\ell}^{\mu}\right)$ is a function of the rapidity $\lambda_{\ell}^{\mu}$ (or the quasimomentum), and $\left\{I_{\ell}^{\mu}\right\}_{\ell, \mu}$ is a set of 
integers or half-odd integers. We assume that the integers $\left\{I_{\ell}^{\mu}\right\}$ satisfy $I_{\ell+1}^{\mu}>I_{\ell}^{\mu}$. We restrict ourselves to the solutions of Eq. (3.1) with rapidities $\lambda_{/}^{\mu}$ all real. It is known that solutions with complex rapidities do exist for many Bethe ansatz solvable systems, but we have not been able to deal with the problem of state counting for such solutions. Below we will address, in several concrete examples, the question of how important are the contributions of solutions with complex rapidities to low-temperature properties of the system.

Subtracting the Bethe ansatz equations (3.1) for adjacent rapidities $\lambda_{\ell}^{\mu}$ gives

$$
\begin{aligned}
L\left[p_{\mu}\left(\lambda_{\ell+1}^{\mu}\right)-p_{\mu}\left(\lambda_{\ell}^{\mu}\right)\right]= & 2 \pi\left(I_{\ell+1}^{\mu}-I_{\ell}^{\mu}\right) \\
& +\sum_{m, \nu}\left[\theta_{\mu \nu}\left(\lambda_{\ell+1}^{\mu}, \lambda_{m}^{\nu}\right)\right. \\
& \left.-\theta_{\mu \nu}\left(\lambda_{\ell}^{\mu}, \lambda_{m}^{\nu}\right)\right] .
\end{aligned}
$$

Following Refs. 18 and 2, we introduce a particle density

$$
\rho_{\mu}\left(\lambda_{\ell}^{\mu}\right)=\frac{1}{L\left(\lambda_{\ell+1}^{\mu}-\lambda_{\ell}^{\mu}\right)}
$$

and a hole density

$$
\rho_{\mu}^{h}\left(\lambda_{\ell}^{\mu}\right)=\frac{M_{\ell}^{\mu}}{L\left(\lambda_{\ell+1}^{\mu}-\lambda_{\ell}^{\mu}\right)},
$$

where $M_{\ell}^{\mu} \geqslant 0$ is the number of the holes in the branches of the Bethe ansatz equations (3.1), i.e., the number $M_{\ell}^{\mu}$ is given by

$$
I_{\ell+1}^{\mu}-I_{\ell}^{\mu}=1+M_{\ell}^{\mu} .
$$

In terms of these densities $\rho_{\mu}$ and $\rho_{\mu}^{h}$, we can rewrite the Bethe ansatz equations (3.2) as

$$
\begin{aligned}
\frac{L}{2 \pi} p_{\mu}^{\prime}\left(\lambda_{i}^{\mu}\right) \Delta \lambda_{i}^{\mu}= & L \rho_{\mu}\left(\lambda_{i}^{\mu}\right) \Delta \lambda_{i}^{\mu}+L \rho_{\mu}^{h}\left(\lambda_{i}^{\mu}\right) \Delta \lambda_{i}^{\mu} \\
& +\frac{1}{2 \pi} \sum_{j, \nu}\left[\theta_{\mu \nu}^{\prime}\left(\lambda_{i}^{\mu}, \lambda_{j}^{\nu}\right) \Delta \lambda_{i}^{\mu}\right] L \rho_{\nu}\left(\lambda_{j}^{\nu}\right) \Delta \lambda_{j}^{\nu},
\end{aligned}
$$

where we denote the derivative of a function $f$ by $f^{\prime}$. Here $L \rho_{\mu}\left(\lambda_{i}^{\mu}\right) \Delta \lambda_{i}^{\mu}$ and $L \rho_{\mu}^{h}\left(\lambda_{i}^{\mu}\right) \Delta \lambda_{i}^{\mu}$ are, respectively, the number of quasiparticles and the number of quasiholes in the interval $\Delta \lambda_{i}^{\mu}$ The total number of possible choices of states with densities $\left\{\rho_{\mu}\right\}$ and $\left\{\rho_{\mu}^{h}\right\}$ is given by

$$
W_{\mathrm{TBA}}=\prod_{i, \mu} \frac{\left\{L\left[\rho_{\mu}\left(\lambda_{i}^{\mu}\right)+\rho_{\mu}^{h}\left(\lambda_{i}^{\mu}\right)\right] \Delta \lambda_{i}^{\mu}\right\} !}{\left[L \rho_{\mu}\left(\lambda_{i}^{\mu}\right) \Delta \lambda_{i}^{\mu}\right] !\left[L \rho_{\mu}^{h}\left(\lambda_{i}^{\mu}\right) \Delta \lambda_{i}^{\mu}\right] !} .
$$

This number $W_{\mathrm{TBA}}$ of states, obtained from the Bethe ansatz equations (3.6), is of exactly the same form as Eq. (2.1) for the number $W$ of states in a generalized ideal gas of exclusons with the statistical interactions given by Eq. (2.2). In fact, we get the equivalence by setting

$$
G_{i}^{\mu}=\frac{L}{2 \pi} p_{\mu}^{\prime}\left(\lambda_{i}^{\mu}\right) \Delta \lambda_{i}^{\mu},
$$

$$
N_{i}^{\mu}=L \rho_{\mu}\left(\lambda_{i}^{\mu}\right) \Delta \lambda_{i}^{\mu}, \quad D_{i}^{\mu}\left(\left\{N_{j}^{\nu}\right\}\right)=L \rho_{\mu}^{h}\left(\lambda_{i}^{\mu}\right) \Delta \lambda_{i}^{\mu},
$$

and

$$
g_{i j}^{\mu \nu}=\delta_{i j} \delta_{\mu \nu}+\frac{1}{2 \pi} \theta_{\mu \nu}^{\prime}\left(\lambda_{i}^{\mu}, \lambda_{j}^{\nu}\right) \Delta \lambda_{i}^{\mu} .
$$

In the thermodynamic limit, the Bethe ansatz equations (3.6) become

$\frac{1}{2 \pi} p_{\mu}^{\prime}(\lambda)=\rho_{\mu}^{h}(\lambda)+\sum_{\nu} \int g^{\mu \nu}\left(\lambda, \lambda^{\prime}\right) \rho_{\nu}\left(\lambda^{\prime}\right) d \lambda^{\prime}$

with the statistical interactions

$$
g^{\mu \nu}\left(\lambda, \lambda^{\prime}\right)=\delta_{\mu \nu} \delta\left(\lambda-\lambda^{\prime}\right)+\frac{1}{2 \pi} \theta_{\mu \nu}^{\prime}\left(\lambda, \lambda^{\prime}\right) .
$$

The key point is that the Bethe ansatz equations (3.11) are equivalent to the equations (2.2) of the statistical interactions in the excluson formalism.

Here we stress once more that in the above we have considered only solutions with real rapidities. Equation (3.12) for statistical interactions refers only to such excitations. But its validity is obviously independent of whether or not these solutions of Bethe ansatz equations are complete. Though right now we do not know how to do state counting for solutions with complex rapidities, we feel it plausible that a slightly more complicated excluson picture may still apply.

\section{THERMODYNAMIC FORMALISM AT FINITE TEMPERATURES}

The thermodynamic formalism for Bethe ansatz solvable models at finite temperatures was first developed by Yang and Yang $^{2}$ for the cases without internal degrees of freedom. This formalism has been justified, e.g., for a one-dimensional Bose gas with repulsive point interaction. ${ }^{19}$ In this section, we briefly review a straightforward generalization of the Yang-Yang thermodynamic formalism in a general setting with internal degrees of freedom, and show that it is actually the same as the generalized ideal gas with the identification (3.8)-(3.10).

In the thermodynamic limit, the number of particles of species $\mu$ per volume is given by

$$
\frac{N_{\mu}}{L}=\int d \lambda \rho_{\mu}(\lambda)
$$

From (3.7), we have the entropy $S=\ln W_{\mathrm{TBA}}$ as

$$
\begin{aligned}
\frac{S}{L}= & \sum_{\mu} \int d \lambda\left\{\left[\rho_{\mu}(\lambda)+\rho_{\mu}^{h}(\lambda)\right] \ln \left[\rho_{\mu}(\lambda)+\rho_{\mu}^{h}(\lambda)\right]\right. \\
& \left.-\rho_{\mu}(\lambda) \ln \rho_{\mu}(\lambda)-\rho_{\mu}^{h}(\lambda) \ln \rho_{\mu}^{h}(\lambda)\right\} .
\end{aligned}
$$

We assume that the total energy per volume is expressed as

$$
\frac{E}{L}=\sum_{\mu} \int d \lambda \rho_{\mu}(\lambda) \epsilon_{\mu}^{0}(\lambda)
$$

in terms of an energy density $\epsilon_{\mu}^{0}$. As a rule, this assumption is always satisfied in Bethe ansatz solvable models. 
The thermodynamic potential $\Omega$ at equilibrium with temperature $T$ can be evaluated by minimizing

$$
\Omega=E-\sum_{\mu} a_{\mu} N_{\mu}-T S
$$

with respect to the variation of the densities $\rho_{\mu}$ and $\rho_{\mu}^{h}$. Here $a_{\mu}$ is the chemical potential.

As a result, we have $\mathrm{e}^{7,2}$

$$
\frac{\Omega}{L}=-\frac{T}{2 \pi} \sum_{\mu} \int d \lambda p_{\mu}^{\prime}(\lambda) \ln \left[1+w_{\mu}^{-1}(\lambda)\right],
$$

where the functions $w_{\mu}$ are determined by the equations

$$
\begin{aligned}
\ln \left[1+w_{\mu}(\lambda)\right]-\sum_{\nu} \int d \lambda^{\prime} g^{\nu \mu}\left(\lambda^{\prime}, \lambda\right) \ln \left[1+w_{\nu}^{-1}\left(\lambda^{\prime}\right)\right] \\
=\frac{\epsilon_{\mu}^{0}(\lambda)-a_{\mu}}{T}
\end{aligned}
$$

The particle densities $\rho_{\mu}$ at equilibrium are determined by the Bethe ansatz equations

$$
\frac{1}{2 \pi} p_{\mu}^{\prime}(\lambda)=\rho_{\mu}(\lambda) w_{\mu}(\lambda)+\sum_{\nu} \int g^{\mu \nu}\left(\lambda, \lambda^{\prime}\right) \rho_{\nu}\left(\lambda^{\prime}\right) d \lambda^{\prime} .
$$

The hole densities are given by $\rho_{\mu}^{h}=\rho_{\mu} w_{\mu}$.

For the thermodynamics reviewed here, the following cautious remark is in order. We have followed the Bethe ansatz method ${ }^{17}$ to reduce the energy eigenvalue problem in the models to solving the so-called Bethe ansatz equations. However, it has not yet been generally proved that all the solutions to the Bethe ansatz equations provide the complete set of energy eigenstates. ${ }^{20}$ In particular, it is known in a number of cases that there are solutions with complex rapidities to the Bethe ansatz equations in addition to those with real rapidities. Though exact thermodynamics must deal with all eigenstates in a complete basis, low-temperature properties of a system, which are the main focus of interest in many situations and in the present paper, might involve only a set of solutions that are not necessarily complete. It has been shown that the ground state at absolute zero always corresponds to a solution with all rapidities real. A sensible question is thus whether the solutions with real rapidities are enough for accounting for low-temperature properties of the system. A way of investigating this problem is to apply the thermodynamics reviewed above to some cases where the exact thermodynamics has been studied by ways that avoid the completeness assumption, and then compare the results to the exact ones. One well-known example of such cases is the Heisenberg spin chain, which we are going to study in the next section.

\section{ISOTROPIC AND ANISOTROPIC HEISENBERG SPIN CHAINS}

In this section, we apply the formalism reviewed in the last section to the isotropic and anisotropic quantum spin-1/2 chains. On one hand, for the spin-1/2 isotropic Heisenberg antiferromagnetic chain at low temperatures, we are able to explicitly demonstrate the magnon excitations (des
Cloizeaux-Pearson-Faddeev-Takhtajan mode ${ }^{21,22}$ ) in the excluson description. On the other hand, we will show that for the isotropic antiferromagnetic chain, the lowtemperature behavior obtained from a generalized ideal gas of doubly degenerate or spin-1/2 excitations with real rapidities does agree with the known exact results ${ }^{23,24}$ which are obtained in other ways without invoking the completeness assumption of string solutions. ${ }^{25}$ Here we stress that for getting the correct results, it is necessary to take into account a double degeneracy of the excitations induced by complex rapidities. Strictly speaking, the solutions of the Bethe Ansatz equations excitations with only real rapidities generally do not properly account for the low-temperature behavior, so other solutions with complex rapidities have to be included. This problem has not been addressed in the previous treatment ${ }^{9}$ of multicomponent systems in one dimension.

\section{A. Spin-1/2 isotropic $(X X X)$ Heisenberg chain}

The Hamiltonian of the spin-1/2 $X X Z$ chain is given by

$$
\mathcal{H}=\sum_{j=1}^{L}\left[S_{j}^{x} S_{j+1}^{x}+S_{j}^{y} S_{j+1}^{y}+\Delta\left(S_{j}^{z} S_{j+1}^{z}-1 / 4\right)\right]
$$

with the periodic boundary condition $\mathbf{S}_{L+1}=\mathbf{S}_{1}$, where $\mathbf{S}_{j}$ is the spin-1/2 operator at site $j$, and $\Delta$ is the anisotropy parameter.

First consider the isotropic $(\Delta=1)$ Heisenberg chain. As is well known, for this model, Bethe ${ }^{17}$ first reduced the eigenvalue problem of the Hamiltonian to solving the so-called Bethe ansatz equations

$$
\begin{gathered}
2 \operatorname{Ltan}^{-1}\left(2 \lambda_{\alpha}\right)=2 \pi I_{\alpha}+2 \sum_{\beta=1}^{M} \tan ^{-1}\left(\lambda_{\alpha}-\lambda_{\beta}\right) \\
(\alpha=1,2, \ldots, M),
\end{gathered}
$$

where $M$ is the number of down spins. The energy eigenvalues are given by

$$
E=-\sum_{\alpha=1}^{M} \frac{2}{1+4 \lambda_{\alpha}^{2}}
$$

Comparing (5.2) with (3.1), we have

$$
p\left(\lambda_{\alpha}\right)=2 \tan ^{-1}\left(2 \lambda_{\alpha}\right)
$$

and

$$
\theta\left(\lambda_{\alpha}, \lambda_{\beta}\right)=2 \tan ^{-1}\left(\lambda_{\alpha}-\lambda_{\beta}\right) .
$$

Further, from (3.12), we get the statistical interactions between magnons as

$$
g\left(\lambda, \lambda^{\prime}\right)=\delta\left(\lambda-\lambda^{\prime}\right)+\frac{1}{\pi} \frac{1}{1+\left(\lambda-\lambda^{\prime}\right)^{2}} .
$$

Yang and Yang proved ${ }^{26}$ that the ground state at $T=0$ indeed corresponds to a solution with all rapidities real, so that it is the same as the ground state of a generalized ideal gas with the above statistical interactions. 


\section{B. Spin-1/2 isotropic Heisenberg chain at low temperatures}

By substituting the results in Sec. V A into (4.5) and (4.6), we get the thermodynamic potential $\Omega$ as

$$
\frac{\Omega}{L}=-\frac{T}{2 \pi} \int_{-\infty}^{\infty} d \lambda \frac{4}{1+4 \lambda^{2}} \ln \left[1+e^{-\epsilon(\lambda) / T}\right],
$$

where $\epsilon=\ln w$ is determined by the equation

$$
\begin{aligned}
\epsilon(\lambda)= & -\frac{2}{1+4 \lambda^{2}}+\frac{T}{\pi} \int_{-\infty}^{\infty} d \lambda^{\prime} \frac{1}{1+\left(\lambda-\lambda^{\prime}\right)^{2}} \\
& \times \ln \left[1+e^{-\epsilon\left(\lambda^{\prime}\right) / T}\right] .
\end{aligned}
$$

Further, from (4.7), we have

$$
\begin{aligned}
\rho(\lambda)\left[1+e^{\epsilon(\lambda) / T}\right]= & \frac{1}{\pi} \frac{2}{1+4 \lambda^{2}}-\frac{1}{\pi} \int_{-\infty}^{\infty} d \lambda^{\prime} \rho\left(\lambda^{\prime}\right) \\
& \times \frac{1}{1+\left(\lambda-\lambda^{\prime}\right)^{2}} .
\end{aligned}
$$

First consider the zero temperature limit. Then (5.8) and (5.9) become

$\epsilon(\lambda)=-\frac{2}{1+4 \lambda^{2}}-\frac{1}{\pi} \int_{-\infty}^{\infty} d \lambda^{\prime} \frac{1}{1+\left(\lambda-\lambda^{\prime}\right)^{2}} \epsilon\left(\lambda^{\prime}\right)$

and

$$
\rho(\lambda)=\frac{1}{\pi} \frac{2}{1+4 \lambda^{2}}-\frac{1}{\pi} \int_{-\infty}^{\infty} d \lambda^{\prime} \rho\left(\lambda^{\prime}\right) \frac{1}{1+\left(\lambda-\lambda^{\prime}\right)^{2}} .
$$

Since these equations are, respectively, linear with respect to the unknown functions $\epsilon$ and $\rho$, one can easily obtain the solutions as

$$
\epsilon(\lambda)=-\frac{\pi}{2 \cosh \pi \lambda}
$$

and

$$
\rho(\lambda)=\frac{1}{2 \cosh \pi \lambda} .
$$

By substituting (5.13) into the right-hand side of (5.9), i.e., by iteration, we get the expression

$$
\rho(\lambda)=\frac{1}{2 \cosh \pi \lambda} \frac{1}{1+e^{\epsilon(\lambda) / T}}
$$

at low temperatures.

To clarify the physical meaning of (5.12) and (5.14), we introduce a new variable $k \in[0, \pi]$ by

$$
\sinh \pi \lambda=-\cot k \text {. }
$$

In terms of $k$, we can rewrite (5.12) and (5.14) as

$$
\epsilon(\lambda)=\varepsilon_{k}=-\frac{\pi}{2}|\sin k|
$$

and

$$
\rho(\lambda) d \lambda=\frac{1}{2 \pi} \frac{d k}{1+e^{\varepsilon_{k} / T}} .
$$

Equation (5.16) implies that holelike excitations have the dressed energy $\varepsilon_{k}^{h}=\pi|\sin k| / 2$, which is nothing but the des Cloizeaux-Pearson-Faddeev-Takhtajan mode. ${ }^{21,22}$ It is known $^{21}$ that the momentum of this mode, $q \in(-\pi, \pi]$, is related to the variable $k$ by

$$
q=\left\{\begin{array}{l}
\pi-k \text { for } 0<q<\pi, \\
-k \text { for }-\pi<q<0 .
\end{array}\right.
$$

This double degeneracy is induced by complex rapidities, ${ }^{24}$ whose net effect is to give spin $1 / 2$ to the excitations. ${ }^{22} \mathrm{~A}$ particular explanation of this degeneracy will be given in the case of the spin-1/2 XY chain below. Equation (5.17) implies that in terms of the dressed energy (5.16), these holelike excitations obey Fermi statistics at low temperatures. In fact, by combining (5.17) with (4.2) and $\rho_{\mu}^{h}=\rho_{\mu} w_{\mu}=\rho_{\mu} e^{\epsilon / T}$, we have the expression of the entropy at low temperatures as

$\frac{S}{L}=-\frac{1}{2 \pi} \int_{-\pi}^{\pi} d q\left\{f\left(\varepsilon_{q}\right) \ln f\left(\varepsilon_{q}\right)+\left[1-f\left(\varepsilon_{q}\right)\right] \ln \left[1-f\left(\varepsilon_{q}\right)\right]\right\}$

with the Fermi distribution function

$$
f(\varepsilon)=\frac{1}{1+e^{\varepsilon / T}}
$$

This result agrees with the free fermion descriptions of the $X X X$ chain. $^{27}$ The entropy (5.19) gives the specific heat $C \cong 2 T / 3$ per volume at low temperatures. The resulting specific heat is just the known exact specific heat. ${ }^{23,24}$ Although we have dropped (string) solutions with complex rapidities to the Bethe ansatz equations, we have been able to get the correct low-temperature behavior by taking into account the double degeneracy of the magnon excitations induced by complex rapidities. Strictly speaking, the contributions to the specific heat from the string solutions cannot be ignored at low temperatures. But the total contribution ${ }^{24}$ appears to be just doubled, due to the spin-1/2 character $^{22}$ of the magnon excitations. This implies that the low-energy excitations such as quasiparticle's can be indeed described by the generalized ideal gas of exclusons with real rapidities only.

\section{Spin-1/2 anisotropic ( $X X Z$ and $X Y)$ chains}

Next consider the spin-1/2 chains (5.1) with anisotropy $-1<\Delta<1$. Then the Bethe ansatz equations are given by ${ }^{26}$

$$
L p\left(\lambda_{\ell}\right)=2 \pi I_{\ell}-\sum_{m=1}^{M} \Theta\left(\lambda_{\ell}, \lambda_{m}\right)
$$

for the states with $M$ down spins. Here

$$
e^{i p(\lambda)}=\frac{e^{i \eta}-e^{\lambda}}{e^{i \eta+\lambda}-1}
$$

and

$\Theta\left(\lambda_{\ell}, \lambda_{m}\right)=-2 \tan ^{-1}\left[(\cot \eta) \tanh \left(\frac{\lambda_{\ell}-\lambda_{m}}{2}\right)\right]$ 
with $\Delta=\cos \eta(0<\eta<\pi)$. The energy eigenvalues are given by

$$
E=-\sum_{\ell=1}^{M}\left[\Delta-\cos p\left(\lambda_{\ell}\right)\right]
$$

Comparing (5.21) with (3.1), and using (3.12), we get the statistical interactions between magnons as

$$
g\left(\lambda, \lambda^{\prime}\right)=\delta\left(\lambda-\lambda^{\prime}\right)+\frac{1}{2 \pi} \frac{\sin 2 \eta}{\cosh \left(\lambda-\lambda^{\prime}\right)-\cos 2 \eta}
$$

Again, the ground state at $T=0$ is a solution with all rapidities real, ${ }^{26}$ so that it coincides with the ground state of a generalized ideal gas with the above statistical interactions.

In the special case of the $X Y$ model, i.e., $\Delta=0$ $(\eta=\pi / 2)$, we have

$$
g\left(\lambda, \lambda^{\prime}\right)=\delta\left(\lambda-\lambda^{\prime}\right)
$$

This implies that quasiparticles (magnons) obey Fermi statistics. Of course, this is consistent with the well-known fact that the spin-1/2 XY chain can be transformed into a system of free spinless fermions in one dimension by using the Jordan-Wigner transformation. ${ }^{28}$

Note that $-(\pi-\eta)<p(\lambda)<(\pi-\eta) \leftrightarrow-\infty<\lambda<+\infty$ from (5.22). In particular, we have $-\pi / 2<p(\lambda)<\pi / 2$ for the $X Y$ chain. Combining this with (5.24), (4.6), and (4.5), we have the thermodynamic potential

$$
\frac{\Omega}{L}=-\frac{T}{2 \pi} \int_{-\pi / 2}^{\pi / 2} d p \ln \{1+\exp [\cos p / T]\}
$$

for the $X Y$ chain. However, this does not coincide with the known exact thermodynamic potential, ${ }^{28}$ which is given by replacing the range of integral (5.27) with $[-\pi, \pi]$. One possible reason for this discrepancy is that we have not taken into account solutions with complex rapidities $\lambda_{\ell}$ to the Bethe ansatz equations (5.21). ${ }^{17}$ But, as is well known, there is no complex rapidity in the $X Y$ chain. ${ }^{28}$ Where does this inconsistency come from? The answer is the following. In the procedure to take the $X Y$ limit $\Delta \rightarrow 0$, we missed a double degeneracy of the magnon excitations. Actually, a more careful treatment taking into account complex rapidities shows this double degeneracy in the $X Y$ limit. $^{29}$ A similar situation occurs also in the $X X X$ chain at low temperatures. ${ }^{24}$ Of course, a much simpler way to get the exact result for the $X Y$ chain is to use the variable $p$ instead of the rapidity $\lambda$. Then one can easily obtain the exact result.

\section{THE HUBBARD CHAIN}

In this section we turn to the Hubbard chain. Our intention is to present an exclusonic description for spin-charge separation, which is known to occur in this model with infinite coupling at zero temperature. ${ }^{30} \mathrm{We}$ will demonstrate spin-charge separation under the following two broader conditions: (i) the on-site Coulomb energy $U$ is large but finite; (ii) the temperature $T$ is finite but sufficiently low compared to the order of the effective exchange $1 / U$.

\section{A. General considerations}

The Hamiltonian of the Hubbard chain is given by

$$
\begin{aligned}
\mathcal{H}= & \sum_{j=1}^{L} \sum_{\sigma=\uparrow, \downarrow}\left(c_{j, \sigma}^{\dagger} c_{j+1, \sigma}+c_{j+1, \sigma}^{\dagger} c_{j, \sigma}\right) \\
& +U \sum_{j=1}^{L} c_{j, \uparrow}^{\dagger} c_{j, \uparrow} c_{j, \downarrow}^{\dagger} c_{j, \downarrow}
\end{aligned}
$$

with on-site Coulomb energy $U$ and periodic boundary conditions $c_{L+1, \sigma}=c_{1, \sigma}(\sigma=\uparrow, \downarrow)$, where $c_{j, \sigma}^{\dagger}$ and $c_{j, \sigma}$ are, respectively, the creation and annihilation operators for an electron with spin $\sigma$ at the site $j$.

For the Hubbard chain, Lieb and $\mathrm{Wu}^{32}$ obtained the Bethe ansatz equations

$$
L k_{i}^{c}=2 \pi I_{i}^{c}+\sum_{\beta=1}^{M} \theta\left(\frac{2}{U} \sin k_{i}^{c}-\lambda_{\beta}^{s}\right) \quad(i=1,2, \ldots, N)
$$

and

$$
\begin{gathered}
-\sum_{j=1}^{N} \theta\left(\lambda_{\alpha}^{s}-\frac{2}{U} \sin k_{j}^{c}\right)=2 \pi I_{\alpha}^{s}-\sum_{\beta=1}^{M} \theta\left(\frac{\lambda_{\alpha}^{s}}{2}-\frac{\lambda_{\beta}^{s}}{2}\right) \\
(\alpha=1,2, \ldots, M)
\end{gathered}
$$

with quasimomenta $\left\{k_{i}^{c}\right\}_{i=1}^{N}$ and rapidities $\left\{\lambda_{\alpha}^{s}\right\}_{\alpha=1}^{M}$ for $(N-M)$ electrons with up spin and $M$ electrons with down spin. Here

$$
\theta(x)=-2 \tan ^{-1}(2 x) .
$$

The energy eigenvalues are given by

$$
E=-2 \sum_{j=1}^{N} \cos k_{j}^{c}
$$

Comparing (6.2) and (6.3) with (3.1), we get

$$
\begin{gathered}
p_{c}\left(k_{i}^{c}\right)=k_{i}^{c}, \\
p_{s}\left(\lambda_{\alpha}^{s}\right)=0, \\
\theta_{c c}\left(k_{i}^{c}, k_{j}^{c}\right)=0, \\
\theta_{c s}\left(k_{i}^{c}, \lambda_{\beta}^{s}\right)=\theta\left(\frac{2}{U} \sin k_{i}^{c}-\lambda_{\beta}^{s}\right), \\
\theta_{s s}\left(\lambda_{\alpha}^{s}, \lambda_{\beta}^{s}\right)=-\theta\left(\frac{\lambda_{\alpha}^{s}}{2}-\frac{\lambda_{\beta}^{s}}{2}\right),
\end{gathered}
$$

and

$$
\theta_{s c}\left(\lambda_{\alpha}^{s}, k_{j}^{c}\right)=\theta\left(\lambda_{\alpha}^{s}-\frac{2}{U} \sin k_{j}^{c}\right) .
$$

Further, from (3.12), we have

$$
g^{c c}\left(k, k^{\prime}\right)=\delta\left(k-k^{\prime}\right),
$$




$$
\begin{aligned}
& g^{c s}(k, \lambda)=-\frac{4}{\pi U} \frac{\cos k}{1+4(2 \sin k / U-\lambda)^{2}}, \\
& g^{s s}\left(\lambda, \lambda^{\prime}\right)=\delta\left(\lambda-\lambda^{\prime}\right)+\frac{1}{\pi} \frac{1}{1+\left(\lambda-\lambda^{\prime}\right)^{2}}
\end{aligned}
$$

and

$$
g^{s c}(\lambda, k)=-\frac{1}{\pi} \frac{2}{1+4(\lambda-2 \sin k / U)^{2}} .
$$

Since in the ground state all $k_{j}^{c}$ and $\lambda_{\alpha}^{s}$ are real, ${ }^{32}$ the ground state is the same as that for a generalized ideal gas with the two species with above statistical interactions. In Ref. 31 it has been shown that solutions with complex quasimomenta and rapidities in the Hubbard model correspond to gapful excitations. Therefore at low temperatures we can ignore thermal activations of such excitations and concentrate on solutions with only real quasimomenta and rapidities.

We note that our results for the Hubbard chain disagree with those in a previous paper ${ }^{14}$ on the same subject. The disagreement comes from the incorrect procedure taken in Ref. 14. In the second equation of Eq. (4) in Ref. 14, the first and fourth terms cancel each other exactly, namely, the fourth term in the right-hand side (RHS), $-\sum_{\beta=1}^{M} \Lambda_{\beta} \delta_{\Lambda_{\alpha}, \Lambda_{\beta}}=-\Lambda_{\alpha}$, cancels the first term. Therefore if one proceeds correctly, the first and fourth terms on the RHS of the second equation of (5) must also cancel. For the same reason, the first and fourth terms on the RHS of (9) should cancel as well. But surprisingly it is not the case in Ref. 14: Two originally canceling terms become eventually noncanceling.

\section{B. Strong coupling expansion for the Hubbard chain}

It is well known that spin and charge degrees of freedom are separated in the strong coupling Hubbard chain at zero temperature. ${ }^{30}$ To explore the possibility of a similar spincharge separation at finite temperatures, we first perform the $1 / U$ expansion for the thermodynamic potential of the Hubbard chain.

To begin with, we will write down the explicit form of the thermodynamic potential $\Omega$ of the Hubbard chain. Substituting the results of the statistical interactions in Sec. VI A into the thermodynamic formulas (4.5) and (4.6) in Sec. IV, we have the thermodynamic potential

$$
\frac{\Omega}{L}=-\frac{T}{2 \pi} \int_{-\pi}^{\pi} d k \ln \left[1+e^{-\epsilon_{c}(k) / T}\right]
$$

for the Hubbard chain, where $\epsilon_{\mu}=\ln w_{\mu}$ are determined by the equations

$$
\begin{aligned}
& \epsilon_{c}(k)+\frac{2 T}{\pi} \int_{-\infty}^{\infty} d \lambda^{\prime} \frac{1}{1+4\left[\lambda^{\prime}-(2 \sin k) / U\right]^{2}} \\
& \times \ln \left[1+e^{-\epsilon_{s}\left(\lambda^{\prime}\right) / T}\right]=-2 \cos k-a_{c}
\end{aligned}
$$

and

$$
\begin{aligned}
\epsilon_{s}(\lambda) & -\frac{T}{\pi} \int_{-\infty}^{\infty} d \lambda^{\prime} \frac{1}{1+\left(\lambda-\lambda^{\prime}\right)^{2}} \ln \left[1+e^{-\epsilon_{s}\left(\lambda^{\prime}\right) / T}\right] \\
& +\frac{4 T}{\pi U} \int_{-\pi}^{\pi} d k \frac{\cos k}{1+4[(2 \sin k) / U-\lambda]^{2}} \ln \left[1+e^{-\epsilon_{c}(k) / T}\right] \\
& =0
\end{aligned}
$$

Now we proceed to the $1 / U$ expansion. When we neglect higher orders than $1 / U$, Eqs. (6.17) and (6.18) become

$$
\begin{gathered}
\epsilon_{c}(k)=-2 \cos k-a_{c}-\frac{2 T}{\pi} \int_{-\infty}^{\infty} d \lambda \frac{1}{1+4 \lambda^{2}} \ln \left[1+e^{-\epsilon_{s}(\lambda) / T}\right], \\
\epsilon_{s}(\lambda)=-\frac{2 J}{1+4 \lambda^{2}}+\frac{T}{\pi} \int_{-\infty}^{\infty} d \lambda^{\prime} \frac{1}{1+\left(\lambda-\lambda^{\prime}\right)^{2}} \\
\times \ln \left[1+e^{-\epsilon_{s}\left(\lambda^{\prime}\right) / T}\right]
\end{gathered}
$$

with the effective exchange integral

$$
J=\frac{2 T}{\pi U} \int_{-\pi}^{\pi} d k \cos k \ln \left[1+e^{-\epsilon_{c}(k) / T}\right] .
$$

Equation (6.20) for $\epsilon_{s}$ corresponds to Eq. (5.8) of the spin$1 / 2$ isotropic Heisenberg chain, although $\epsilon_{s}$ and $\epsilon_{c}$ couple to each other.

Similarly, we have the equations for the charge density $\rho_{c}$ and the spin density $\rho_{s}$ as

$$
\rho_{c}(k)\left[1+e^{\epsilon_{c}(k) / T}\right]=\frac{1}{2 \pi}+\frac{1}{\pi U} \int_{-\infty}^{\infty} d \lambda \rho_{s}(\lambda) \frac{4}{1+4 \lambda^{2}} \cos k
$$

and

$$
\begin{aligned}
\rho_{s}(\lambda)\left[1+e^{\epsilon_{s}(\lambda) / T}\right]= & \frac{1}{2 \pi} \frac{N}{L} \frac{4}{1+4 \lambda^{2}}-\frac{1}{\pi} \int_{-\infty}^{\infty} d \lambda^{\prime} \rho_{s}\left(\lambda^{\prime}\right) \\
& \times \frac{1}{1+\left(\lambda-\lambda^{\prime}\right)^{2}}
\end{aligned}
$$

from (4.7), where $N$ is the number of electrons. Here we have neglected higher orders than $1 / U$. Comparing (6.23) with (5.9) of the spin-1/2 isotropic Heisenberg chain, we obtain that the number $\rho_{s}$ of down spins per volume in the Hubbard chain is proportional to the number of electrons per volume.

Clearly, from (6.19)-(6.23), we conclude that the degrees of freedom of spin and charge parts still couple to each other at finite temperatures.

\section{Spin-charge separation in the Hubbard chain at low temperatures}

In this section, we will demonstrate that spin-charge separation occurs in the strong coupling Hubbard chain at low temperatures.

Consider the case with low temperatures $T \ll 1 / U$. That is to say, temperatures are sufficiently low compared to the effective exchange $J(6.21)$ which behaves as 


$$
J \cong \frac{2}{\pi U} \int_{D_{-}} d k \cos k\left[-\epsilon_{c}(k)\right]
$$

at low temperatures. Here $D_{-}=\left\{k \mid \epsilon_{c}(k)<0\right\}$. Then we have $\epsilon_{s}=O(1 / U)$ from $(6.20)$.

Note that $\epsilon_{c}(6.19)$ can be rewritten as

$$
\boldsymbol{\epsilon}_{c}(k)=\boldsymbol{\epsilon}_{c}^{(0)}(k)+\boldsymbol{\epsilon}_{c}^{(1)}
$$

with

$$
\epsilon_{c}^{(0)}(k)=-2 \cos k-a_{c}
$$

and

$$
\epsilon_{c}^{(1)}=-\frac{2 T}{\pi} \int_{-\infty}^{\infty} \frac{d \lambda}{1+4 \lambda^{2}} \ln \left[1+e^{-\epsilon_{s}(\lambda) / T}\right] .
$$

Here $\epsilon_{c}^{(1)}=O(1 / U)$ due to the above $\epsilon_{s}=O(1 / U)$.

From this observation, we divide the integral of (6.16) into two parts as

$$
\frac{\Omega}{L}=I_{+}+I_{-},
$$

where

$$
I_{ \pm}=-\frac{T}{2 \pi} \int_{D_{ \pm}} d k \ln \left[1+e^{-\epsilon_{c}(k) / T}\right],
$$

and the ranges of integration are $D_{+}=\left\{k \mid \epsilon_{c}^{(0)}(k)\right.$ $\left.\left.+\epsilon_{c}^{(1)}\right\}>0\right\}$ and $D_{-}=\left\{k \mid \epsilon_{c}^{(0)}(k)+\epsilon_{c}^{(1)}<0\right\}$. Since $\epsilon_{c}^{(0)} \gg \epsilon_{c}^{(1)}=O(1 / U)$ and $T \ll 1 / U, I_{ \pm}$(6.29) are given up to the order $1 / U$ as

$$
I_{+} \cong-\frac{T}{2 \pi} \int_{D_{+}} d k \ln \left[1+e^{-\epsilon_{c}^{(0)}(k) / T}\right]
$$

and

$$
\begin{aligned}
I_{-}= & \frac{1}{2 \pi} \int_{D_{-}} d k\left\{\epsilon_{c}^{(0)}(k)+\epsilon_{c}^{(1)}\right\} \\
& -\frac{T}{2 \pi} \int_{D_{-}} d k \ln \left[1+e^{\left\{\epsilon_{c}^{(0)}(k)+\epsilon_{c}^{(1)}\right\} / T}\right] \\
\cong & \frac{N}{L} \epsilon_{c}^{(1)}+\frac{1}{2 \pi} \int_{D_{-}} d k \epsilon_{c}^{(0)}(k) \\
& -\frac{T}{2 \pi} \int_{D_{-}} d k \ln \left[1+e^{\epsilon_{c}^{(0)}(k) / T}\right] \\
= & \frac{N}{L} \epsilon_{c}^{(1)}-\frac{T}{2 \pi} \int_{D_{-}} d k \ln \left[1+e^{-\epsilon_{c}^{(0)}(k) / T}\right],
\end{aligned}
$$

where we have used the relation $\int_{D_{-}} d k / 2 \pi \cong N / L$ which is derived from (6.22) at low temperatures and for large $U$. Thus we have

$$
\begin{aligned}
\frac{\Omega}{L} & \cong-\frac{T}{2 \pi} \int_{-\pi}^{\pi} d k \ln \left[1+e^{-\epsilon_{c}^{(0)}(k) / T}\right]+\frac{N}{L} \epsilon_{c}^{(1)} \\
& =\frac{\Omega_{c}}{L}-\frac{N}{L} \frac{2 T}{\pi} \int_{-\infty}^{\infty} \frac{d \lambda}{1+4 \lambda^{2}} \ln \left[1+e^{-\epsilon_{s}(\lambda) / T}\right]
\end{aligned}
$$

for the thermodynamic potential $\Omega(6.16)$ up to the order $1 / U$, where we have used (6.27), and the thermodynamic potential $\Omega_{c}$ of the charge part is given by

$$
\frac{\Omega_{c}}{L}=-\frac{T}{2 \pi} \int_{-\pi}^{\pi} d k \ln \left[1+e^{-\epsilon_{c}^{(0)}(k) / T}\right] .
$$

Thus the thermodynamic potential $\Omega$ of the Hubbard chain can be written as the sum of the charge and the spin parts,

$$
\frac{\Omega}{L} \cong \frac{\Omega_{c}}{L}+\frac{N}{L} \frac{\Omega_{s}}{N},
$$

where

$$
\frac{\Omega_{s}}{N}=-\frac{T}{2 \pi} \int_{-\infty}^{\infty} d \lambda \frac{4}{1+4 \lambda^{2}} \ln \left[1+e^{-\epsilon_{s}(\lambda) / T}\right]
$$

which is identical to the thermodynamic potential (5.7) of the spin-1/2 isotropic Heisenberg chain. Equation (6.34) is an indication of charge-spin separation.

\section{EXACTLY SOLVABLE MODEL IN HIGHER DIMENSIONS}

So far we have discussed models in one dimension to which the Bethe ansatz approach is applicable. In two dimensions, it is known that the quasiparticles of the fractional quantum Hall liquid are anyons. They can also be considered to be exclusons. ${ }^{3}$ In this section we present an example of mutual exclusion between different species in an exactly solvable model in higher dimensions, which exhibits chargespin separation under certain circumstances. This clearly shows that exclusion statistics is conceptually different from anyon statistics, whose existence requires two (spatial) dimensions.

Recently two of us ${ }^{13}$ and Baskaran ${ }^{33}$ have proposed a model of interacting electrons that can be solved exactly in any dimensions. The Hamiltonian is

$$
\begin{aligned}
H= & -\sum_{\langle i, j\rangle} c_{i, \sigma}^{\dagger} c_{j, \sigma}+\text { H. c. } \\
& +\frac{U}{L^{d}} \sum_{i, j, \ell, m} \delta_{i+j, \ell+m} c_{i, \uparrow}^{\dagger} c_{j, \uparrow} c_{\ell, \downarrow}^{\dagger} c_{m, \downarrow} \\
& -\sum_{i, \sigma}\left(\mu+\sigma \mu_{0} h\right) c_{i, \sigma}^{\dagger} c_{i, \sigma},
\end{aligned}
$$

where $\langle i, j\rangle$ represents nearest neighbors in $d$ dimensions, and $L^{d}$ is the total number of lattice sites, $\mu$ the chemical potential, $\mu_{0}$ the magnetic moment, and $h$ the external magnetic field.

This model is unrealistic in the sense that the interaction term with coefficient $U$ is of infinite range in real space and of strength independent of distance. (Note that the noninteracting electron models are even more unrealistic since they neglect the long-range Coulomb force.) It, however, has the attractive feature of being exactly solvable. In fact, it can be easily diagonalized for each $k$ in momentum space. All the properties, including the thermodynamic quantities, were ob- 


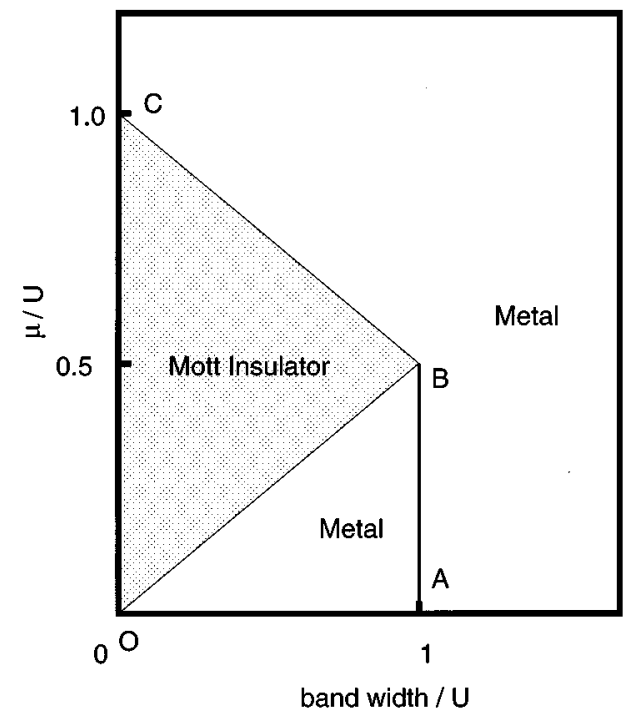

FIG. 1. Phase diagram of the exactly solvable model in higher dimensions at $T=0$.

tained in Ref. 13. Furthermore, it is remarkable that this model exhibits a number of important features of correlated electron problems in spite of its simplicity and unrealistic nature. It exhibits, for example, the Mott metal-insulator transition that was stressed by two of us ${ }^{13}$ and Continentino and Coutinho-Filho. ${ }^{34}$

The zero-temperature phase diagram of this model in any dimensions is shown in Fig. 1. It has both the fixed-density and density-driven Mott transitions. These transitions in general may be in different universality classes. ${ }^{35,36}$ The critical exponents of the two types of transitions are, however, the same in the present model and they seem to be in the same universality class. ${ }^{34}$ In the zero-temperature phase diagram Fig. 1, the region $O B C$ is a Mott insulator phase with halffilled band. The rest are metallic phases. In the region $O A B C$, a double occupancy is prohibited and, as we will show, the system can be described by an excluson picture. There is a Fermi surface of the excluson gas in the region $O A B$, and on the phase transition line $O B$ is a quantum phase transition of the excluson gas. On the other hand, the excluson description breaks down on the phase transition line $B C$. The region outside $O A B C$ is a metallic phase which is described by two species of fermions (spin up and spin down electrons), as it should be.

Let us concentrate on the region $O A B$. Assume that $U$ is large and $T$ is low, so that $U$ is much larger than both $T$ and the bandwidth. ${ }^{37}$ Under these conditions, there is no activation of doubly occupied states; therefore there are only three states $(0,1$, and 2$)$ for each momentum $k$. In the state 0 there is no electron, the state 1 there is an electron with spin up, and in the state 2 an electron with spin down. Let us denote the number of charges as $N_{c}$ and the number of magnons (number with spin down) as $N_{s}$. We regard $N_{c}$ and $N_{s}$ as independent variables (spin-charge separation). By definition, the state 0 has $N_{c}=0$ and $N_{s}=0$, the state $1 N_{c}=1$ and $N_{s}=0$ and the state $2 N_{c}=1$ and $N_{s}=1$ (see Table I). Then from (2.2), we easily derive

$$
G_{c}=1, \quad G_{s}=0,
$$

TABLE I. Electronic states and spin-charge labels.

\begin{tabular}{cccc}
\hline \hline Electronic state & Label & $N_{c}:$ Charge & $N_{s}:$ Magnon \\
\hline 0 & 0 & 0 & 0 \\
$\uparrow$ & 1 & 1 & 0 \\
$\downarrow$ & 2 & 1 & 1 \\
\hline \hline
\end{tabular}

and

$$
\left[\begin{array}{ll}
g_{c c} & g_{c s} \\
g_{s c} & g_{s s}
\end{array}\right]=\left[\begin{array}{cc}
1 & 0 \\
-1 & 1
\end{array}\right]
$$

It is easy to verify that the condition for the ideal excluson gas (2.4) is satisfied, so that the system with doubly occupied states suppressed can be described as a generalized ideal gas with two (charge and magnon) species with the statistical interaction given by (7.3). Note the nontrivial value -1 for mutual statistics $g_{s c}$; i.e., the presence of a charge can create a magnon state, though there is no bare available singlemagnon state $\left(G_{s}=0\right)$ when there is no charge. It is straightforward to check that the thermodynamics of the generalized ideal excluson gas obtained from Eqs. (2.6) and (2.8) is identical to the result of Ref. 13 in the low-temperature limit.

Indeed, in the present case, the species index $\mu=c, s$, and the state index $j$ is the momentum $k$ in $d$-dimensional space. Equation (2.8) now takes the form

$\Omega=-T \sum_{\mu} \int \frac{d^{d} k}{(2 \pi)^{d}} \ln \left[\frac{1-n_{\mu}(k)-\sum_{\nu} g_{\mu \nu} n_{\nu}(k)}{1-\sum_{\nu} g_{\mu \nu} n_{\nu}(k)}\right]$,

where $n_{\mu}(k)$ is the occupation number distribution function of the charge $(\mu=c)$ or spin $(\mu=s)$ excitations in $k$ space. From Eqs. (2.5) and (2.6), we have

$$
n_{c}(k)\left[1+w_{c}(k)\right]=1, \quad n_{s}(k)\left[1+w_{s}(k)\right]=n_{c}(k),
$$

where the statistics matrix (7.3) has been used, and $w_{c}(k)$ and $w_{s}(k)$ satisfy

$$
\begin{gathered}
w_{c}(k) \frac{1+w_{s}(k)}{w_{s}(k)}=e^{\left(\epsilon_{c}(k)-\mu_{c}\right) / T}, \\
w_{s}(k)=e^{\left(\epsilon_{s}(k)-\mu_{s}\right) / T},
\end{gathered}
$$

where $\epsilon_{c}(k)=-2 \Sigma_{\alpha=1}^{d} \cos \left(k_{\alpha}\right), \epsilon_{s}=2 \mu_{0} h$ (the energy of spin excitation, which is actually measured relative to the energy of spin-up electrons), and

$$
\mu_{c}=\mu+\mu_{0} h, \quad \mu_{s}=0 .
$$

Thus

$$
w_{c}(k)=\frac{e^{\left[\epsilon_{c}(k)-\mu_{c}\right] / T}}{1+e^{-\left(\epsilon_{s}(k)-\mu_{s}\right] / T}} .
$$

Substituting Eqs. (7.9), (7.7), and (7.5) into (7.4), we obtain 


$$
\begin{aligned}
\Omega & =-T \int \frac{d^{d} k}{(2 \pi)^{d}} \ln \left[1+\left(e^{\mu_{0} h / T}+e^{-\mu_{0} h / T}\right) e^{\left(\mu_{c}-\epsilon_{c}\right) / T}\right] \\
& =-T \int \frac{d^{d} k}{(2 \pi)^{d}} \ln \left[1+e^{-\left(\epsilon_{1}(k)-\mu\right) / T}+e^{-\left(\epsilon_{2}(k)-\mu\right) / T}\right],
\end{aligned}
$$

where $\epsilon_{1}(k)=\epsilon_{c}(k)-\mu_{0} h$ and $\epsilon_{2}(k)=\epsilon_{c}(k)+\mu_{0} h$ are the energies of spin-up and spin-down electrons, respectively. Equation (7.10) is nothing but the result of Ref. 13 in the low-temperature limit. (Remember that here we consider the case with large $U$ and low $T$, so that doubly occupied states are suppressed.)

Here we emphasize that the concept of spin-charge separation is crucial. The effect that spin and charge excitations are not actually independent of each other have been taken

* Present address: Department of Applied Physics, University of Tokyo, 7-3-1, Hongo, Bunkyo-ku, Tokyo 113, Japan; electronic address: hatsugai@coral.t.u-tokyo.ac.jp

†Electronic address: koma@riron.gakushuin.ac.jp

${ }^{1}$ See, e.g., Fractional Statistics and Anyon Superconductivity, edited by F. Wilczek (World Scientific, Singapore, 1989), and references therein.

${ }^{2}$ C. N. Yang and C. P. Yang, J. Math. Phys. 10, 1115 (1969).

${ }^{3}$ F. D. M. Haldane, Phys. Rev. Lett. 66, 937 (1991).

${ }^{4}$ F. Wilczeck, Phys. Rev. Lett. 48, 1144 (1982).

${ }^{5}$ B. I. Halperin, Phys. Rev. Lett. 52, 1583 (1984).

${ }^{6}$ Y. S. Wu, Phys. Rev. Lett. 52, 2103 (1984).

${ }^{7}$ Y. S. Wu, Phys. Rev. Lett. 73, 922 (1994).

${ }^{8}$ D. Bernard and Y. S. Wu, in Proceedings of the Sixth Nankai Workshop, Tianjin, China, 1994, edited by by Mo-lin Ge and Y. S. Wu (World Scientific, Singapore, 1995).

${ }^{9}$ T. Fukui and N. Kawakami, Phys. Rev. B 51, 5239 (1995).

${ }^{10}$ Y. S. Wu and Yue Yu, Phys. Rev. Lett. 75, 890 (1995).

${ }^{11}$ D. C. Mattis and E. H. Lieb, J. Math. Phys. 6, 304 (1965).

${ }^{12}$ F. Calogero, J. Math. Phys. 10, 2191 (1969); 10, 2197 (1969); B. Sutherland, ibid. 12, 251 (1971).

${ }^{13}$ Y. Hatsugai and M. Kohmoto, J. Phys. Soc. Jpn. 61, 2056 (1992).

${ }^{14}$ K. Byczuk and J. Spalek, Phys. Rev. B 50, 11403 (1995).

${ }^{15}$ Y. S. Wu, Phys. Rev. Lett. 53, 111 (1984).

${ }^{16}$ See, e.g., B. Sutherland, in Exactly Solvable Problems in Condensed Matter and Relativistic Field Theory, edited by B. S. Shastry, S. S. Jha, and V. Singh, Lecture Notes in Physics Vol. 242 (Springer, Berlin, 1985), p. 1.

${ }^{17}$ H. A. Bethe, Z. Phys. 71, 205 (1931). care of by the statistical interaction or mutual statistics between them in the present formulation. It seems to us that similar situations may happen in other strongly correlated systems that exhibit charge-spin separation in higher dimensions. We finally note that our treatment for the exactly solvable models in higher dimensions disagrees with that in a previous paper ${ }^{14}$ on the same subject.

\section{ACKNOWLEDGMENTS}

This work was supported by a Grant-in-Aid from the Ministry of Education, Science and Culture of Japan. The work of Y.S. Wu was also supported in part by U.S. NSF Grant No. PHY-9309458. He is also grateful for warm hospitality of the Institute for Solid State Physics, University of Tokyo, during his visits.

${ }^{18}$ E. H. Lieb and W. Liniger, Phys. Rev. 130, 1605 (1963).

${ }^{19}$ T. C. Dorlas, J. T. Lewis, and J. V. Pulé, Commun. Math. Phys. 124, 365 (1989); T. C. Dorlas, ibid. 154, 347 (1993).

${ }^{20}$ T. Koma and H. Ezawa, Prog. Theor. Phys. 78, 1009 (1987).

${ }^{21}$ J. des Cloizeaux and J. J. Pearson, Phys. Rev. 128, 2131 (1962).

${ }^{22}$ L. D. Faddeev and L. Takhtajan, Phys. Lett. 85A, 375 (1981); J. Sov. Math. 24, 241 (1984).

${ }^{23}$ M. Takahashi, Prog. Theor. Phys. 50, 1519 (1973); K. Nomura and M. Yamada, Phys. Rev. B 43, 8217 (1991); J. Suzuki, Y. Akutsu, and M. Wadati, J. Phys. Soc. Jpn. 50, 2667 (1990); C. Destri and H. J. de Vega, Phys. Rev. Lett. 69, 2313 (1992).

${ }^{24}$ H. M. Babujian, Nucl. Phys. B215 [FS7], 317 (1983).

${ }^{25}$ T. Koma, Prog. Theor. Phys. 78, 1213 (1987); 81, 783 (1989).

${ }^{26}$ C. N. Yang and C. P. Yang, Phys. Rev. 150, 321 (1966).

${ }^{27}$ T. Yamada, Prog. Theor. Phys. 41, 880 (1969); I. Affleck, Phys. Rev. Lett. 56, 746 (1986).

${ }^{28}$ E. Lieb, T. Schultz, and D. Mattis, Ann. Phys. (N.Y.) 16, 407 (1961); S. Katsura, Phys. Rev. 127, 1508 (1962); 129, 2835 (1963).

${ }^{29}$ M. Takahashi and M. Suzuki, Prog. Theor. Phys. 48, 2187 (1972).

${ }^{30}$ M. Ogata and H. Shiba, Phys. Rev. B 41, 2326 (1990).

${ }^{31}$ F. Woynarovich, J. Phys. C 15, 85 (1982); 15, 97 (1982).

${ }^{32}$ E. H. Lieb and F. Y. Wu, Phys. Rev. Lett. 20, 1445 (1968).

${ }^{33}$ G. Baskaran, Int. J. Mod. Phys. Lett. B 5, 643 (1991).

${ }^{34}$ M. A. Continentino and M. D. Coutinho-Filho, Solid State Commun. 90, 619 (1994).

${ }^{35}$ M. A. Continentino, Phys. Rep. 239, 179 (1994).

${ }^{36}$ M. P. A. Fisher, P. B. Weichman, G. Grinstein, and D. Fisher, Phys. Rev. B 40, 546 (1989).

${ }^{37}$ J. Spalek and W. Wójcik, Phys. Rev. B 37, 1532 (1988). 